\title{
Combination therapy with corticosteroid and mycophenolate mofetil in a case of refractory cardiac sarcoidosis
}

\author{
Noriko Kikuchi (MD) ${ }^{a}$, Shinichi Nunoda (MD, PhD, FJCC) $)^{\text {b** }}$, Naoki Serizawa (MD, PhD) \\ Atsushi Suzuki (MD, PhD) a , Tsuyoshi Suzuki (MD, PhD)a , Kenji Fukushima (MD, PhD ${ }^{c}$, \\ Kenta Uto $(\mathrm{MD}, \mathrm{PhD})^{\mathrm{d}}$, Tsuyoshi Shiga $(\mathrm{MD}, \mathrm{PhD}, \mathrm{FJCC})^{\mathrm{a}}$, Morio Shoda $(\mathrm{MD}, \mathrm{PhD})^{\mathrm{a}}$, \\ Nobuhisa Hagiwara (MD, PhD, FJCC) ${ }^{\mathrm{a}}$
}

a Department of Cardiology, Tokyo Women's Medical University, Tokyo, Japan

${ }^{\mathrm{b}}$ Division of Severe Heart Failure, Tokyo Women's Medical University Graduate School of Medicine, Tokyo, Japan

${ }^{\mathrm{c}}$ Department of Diagnostic Imaging and Nuclear Medicine, Tokyo Women's Medical University, Tokyo, Japan

${ }^{\mathrm{d}}$ Department of Pathology, Tokyo Women's Medical University, Tokyo, Japan

\section{A R T I C L E I N F O}

\section{Article history:}

Received 30 October 2015

Received in revised form 11 December 2015

Accepted 24 December 2015

\section{Keywords:}

Cardiac sarcoidosis

Mycophenolate mofetil

Corticosteroid

Ventricular tachycardia

\begin{abstract}
A B S T R A C T
Management of cardiac sarcoidosis (CS) can be challenging. The first-line therapy for this condition is corticosteroids, but other immunosuppressive agents are sometimes co-administered to reduce the dosage of corticosteroid and to thereby avoid steroid-induced adverse effects or to increase its therapeutic efficacy. Mycophenolate mofetil (MMF) is a prodrug of mycophenolic acid, an inhibitor of inosine monophosphate dehydrogenase that acts more selectively on $\mathrm{T}$ and $\mathrm{B}$ lymphocytes when compared with azathioprine. A 40-year-old man was diagnosed with CS after presenting with ventricular fibrillation. His left ventricular ejection fraction was severely reduced (30\%), and cardiac positron emission tomography (PET) showed abnormal uptake of ${ }^{18} \mathrm{~F}$-fluorodeoxyglucose. A cardioverter-defibrillator was implanted and prednisolone $(30 \mathrm{mg} /$ day $)$ was administered. He was re-admitted with recurrent sustained ventricular tachycardia and a positive PET finding despite a 5 -month course of prednisolone, and MMF (1000 mg/day) was administered. Six months later, he had not required re-hospitalization for heart failure or arrhythmia. We conclude that combination therapy with MMF and corticosteroids is useful for refractory CS.

<Learning objective: Management of cardiac sarcoidosis (CS) can be challenging. Although some immunosuppressive agents are co-administered to reduce the dosage of corticosteroids or to intensify the effect of corticosteroids, the optimal combination regimen has not yet been established. This case report shows that combination therapy with corticosteroid and mycophenolate mofetil was useful for CS that was refractory to corticosteroid monotherapy.>
\end{abstract}

(c) 2016 Japanese College of Cardiology. Published by Elsevier Ltd. All rights reserved.

\section{Introduction}

Sarcoidosis is a granulomatous disease that can affect multiple organs, including the lung, liver, nerve, skin, and heart [1]. Cardiac involvement can also occur and manifest as arrhythmias with ventricular tachyarrhythmia and sudden death or heart failure [2]. Cardiac magnetic resonance (CMR) and positron emission tomography (PET) can be used to diagnose cardiac sarcoidosis (CS)

\footnotetext{
* Corresponding author at: Division of Severe Heart Failure, Tokyo Women's Medical University Graduate School of Medicine, 8-1 Kawada-cho, Shinjuku-ku, Tokyo 1628666, Japan. Tel.: +8133353 8111; fax: +81333560441.

E-mail address: ie9s-nnd@asahi-net.or.jp (S. Nunoda).
}

at an early time point [3]. Moreover, PET may be used to assess the activity of CS [4].

While early diagnosis and early treatment of CS may improve outcomes, treatment can be challenging. The main goal of treatment for sarcoidosis is to control and suppress the inflammation and activity of the disease. Corticosteroids comprise the first line of therapy [1]. However, the use of corticosteroids is frequently accompanied by serious adverse effects, such as gastric ulcers, osteoporosis, diabetes, and infectious diseases. Other immunosuppressive agents (e.g. methotrexate, azathioprine, leflunomide) are sometimes added to reduce the dosage of corticosteroids or to intensify therapy, but some patients do not respond to or tolerate these agents [5]. Mycophenolate mofetil (MMF) is a prodrug of mycophenolic acid, an inhibitor of inosine monophosphate 
A

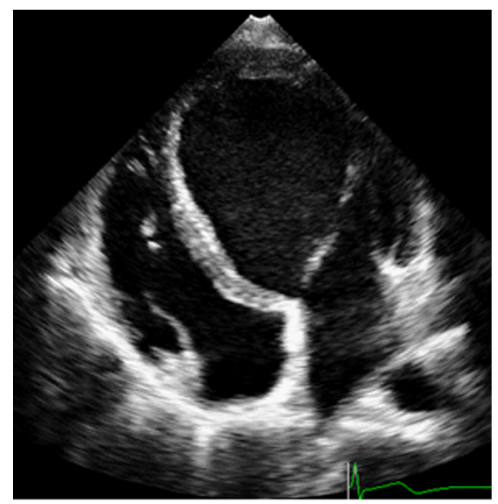

B

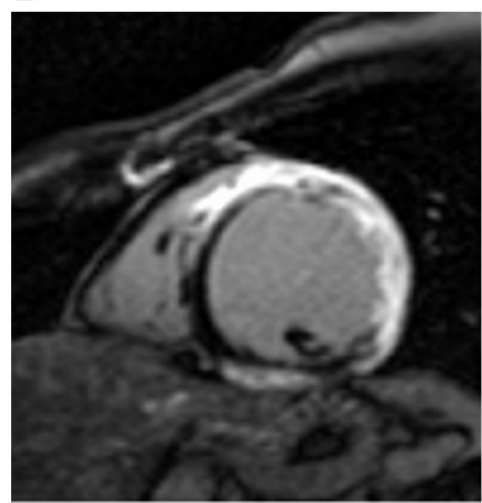

C

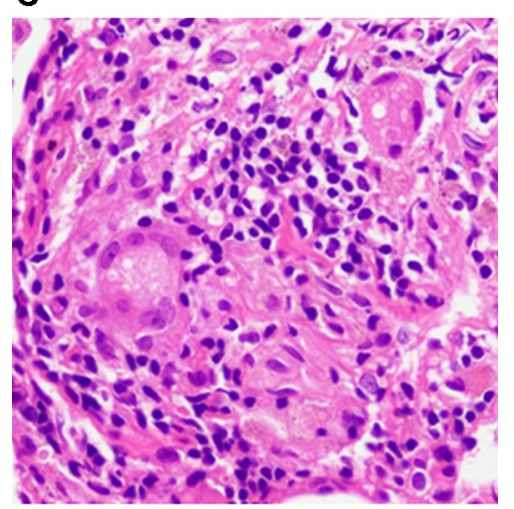

Images leading to the diagnosis of cardiac sarcoidosis. (A) Transthoracic echocardiography shows a left ventricle (LV) ejection fraction of $30 \%$ and LV dilation. (B) Cardiovascular magnetic resonance shows the presence of a late enhancement of gadolinium contrast involving the interventricular septum and LV anteriorinferior-lateral-posterior walls. The pattern of enhancement involves the mid-myocardium and epicardium. (C) Trans-bronchial lung biopsy shows noncaseating epithelioid granuloma.

dehydrogenase [6], and is a key immunosuppressive agent used in solid organ transplant recipients [7]. A retrospective study demonstrated the efficacy and safety of MMF with corticosteroids in 10 patients with pulmonary sarcoidosis [8]. However, data regarding the use of MMF in cardiac sarcoidosis are limited. The present report describes a case of steroid-resistant CS that was successfully treated with combination therapy of MMF and corticosteroids.

\section{Case report}

A 40-year-old man was admitted due to ventricular fibrillation. He was resuscitated using an automated external defibrillator. On admission, he was alert and his electrocardiogram showed ventricular tachycardia. His coronary angiography revealed neither stenosis nor occlusion, and his echocardiogram showed a left ventricular (LV) ejection fraction (EF) of $30 \%$ and LV dilation (LV enddiastolic diameter, $61 \mathrm{~mm}$ ) (Fig. 1A). Laboratory testing showed a serum angiotensin-converting enzyme (ACE) level of $12.5 \mathrm{U} / \mathrm{L}$ (normal range: 8.3-21.4 U/L). CMR showed a significantly enlarged left ventricle and severe diffuse hypokinesis. Late gadolinium enhancement (LGE) revealed extensive epicardial hyper-enhancement in the anterior, lateral, and inferior walls (Fig. 1B). An endomyocardial biopsy did not demonstrate granulomatous inflammation, but a trans-bronchial lung biopsy (Fig. 1C) showed sarcoidosis.

An implantable cardioverter-defibrillator (ICD) was placed, and medical therapy (including $20 \mathrm{mg}$ of carvedilol) was initiated. Cardiac ${ }^{18}$ F-fluorodeoxyglucose (FDG) PET was performed to evaluate for myocardial inflammation and showed significant FDG uptake in the wall that corresponded to the LGE area. The maximal standardized uptake value (SUV max) was 6.01 in the anterolateral wall (Fig. 2A and B). No active inflammation was observed in other organs (Fig. 2A).

One month later, the patient was re-admitted to the hospital with frequent but appropriate discharges of his ICD. His laboratory data on admission demonstrated high brain natriuretic peptide $(1000 \mathrm{pg} / \mathrm{ml})$, and prednisone (30 mg/day) and amiodarone therapy was initiated. Four months after starting prednisone, he was readmitted with recurrent ventricular tachycardia (VT). His serum ACE level remained low (6-7 U/L). Follow-up FDG-PET study revealed positive findings (SUV max =4.46) despite an 8-month course of prednisolone (Fig. 3).

To intensify the therapy, we increased the amount of corticosteroid, but VT storm occurred again. MMF (1000 mg; CellCept ${ }^{\circledR}$; Roche Laboratories, Basel, Switzerland) was added to corticosteroid in order to intensify the immunosuppressive effect. Using blood concentration monitoring, we adjusted the dosage of MMF to a target of $2.0 \mu \mathrm{g} / \mathrm{ml}$, and the frequency of ICD discharges
A

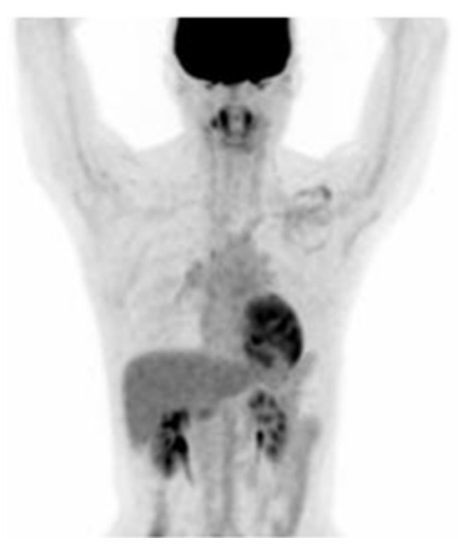

B

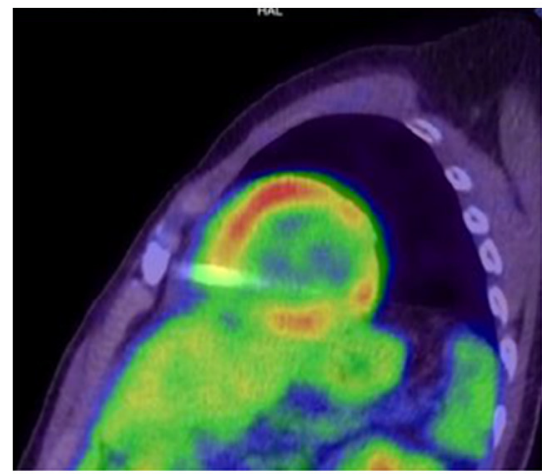




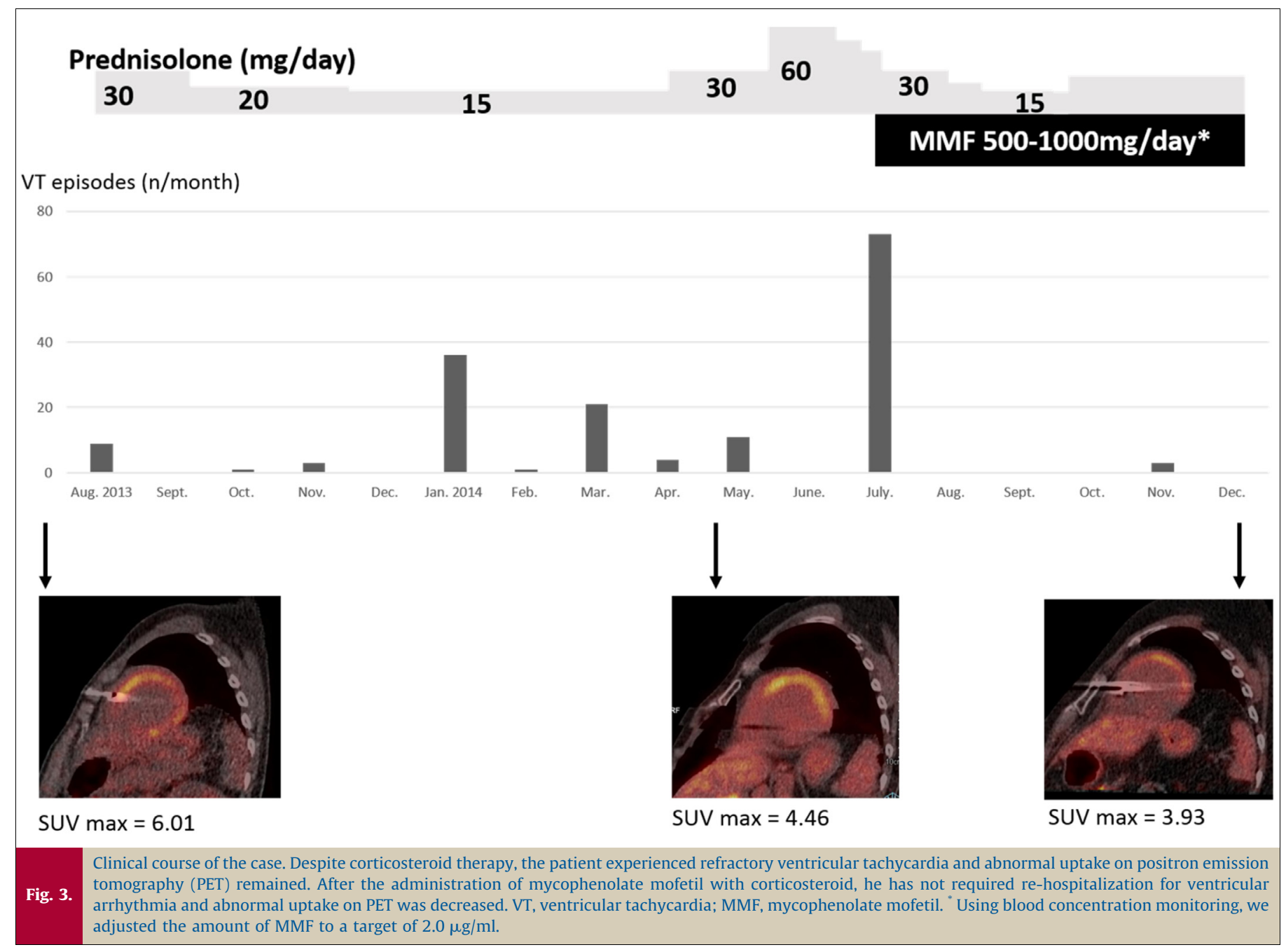

for VT gradually decreased. Obvious adverse effects of MMF, such as gastrointestinal symptoms and leukopenia, did not occur. At 6 months after the initiation of MMF, the patient has not required re-hospitalization for either heart failure or arrhythmia. The PET SUV value decreased slightly (SUV max $=3.93$ ) (Fig. 3), and his cardiac function remained impaired $(\mathrm{EF}=25 \%)$.

\section{Discussion}

The report describes the use of combination therapy of corticosteroids and MMF in a case of refractory CS. Brill et al. reported that the addition of MMF to corticosteroid in 10 patients with chronic pulmonary sarcoidosis allowed for a significant reduction in the dosage of corticosteroids while simultaneously maintaining lung function. They also reported that the addition of MMF was safe and well tolerated [8].

Corticosteroids comprise the first line of therapy for sarcoidosis. Other immunosuppressive agents, such as methotrexate and azathioprine, are sometimes co-administered either to reduce the dosage of corticosteroid (to decrease the risk of steroid-related adverse effects) or to intensify its efficacy [5]. Due to its efficacy, methotrexate is one of the most commonly used corticosteroidsparing therapies for sarcoidosis [5,9], and the drug can be given orally or subcutaneously. Since methotrexate is cleared by the kidneys, monitoring of renal function is advised, and adjustment of the methotrexate dose is needed in patients with renal insufficiency (i.e. serum creatinine $>1.5 \mathrm{mg} / \mathrm{dl}$ ). Azathioprine, which has been used with other medications to prevent transplant rejection, has recently been studied in the management of sarcoidosis. However, azathioprine increases the risk of infection [9]. On the other hand, MMF is a prodrug of mycophenolic acid, an inhibitor of inosine monophosphate dehydrogenase, and acts more selectively on $\mathrm{T}$ and $\mathrm{B}$ lymphocytes when compared with azathioprine $[7,10]$. The rapid response and more selective inhibition of lymphocytes by MMF may be important advantages over other immunosuppressive agents [8]. Indeed, the use of MMF is thought to have more immunological efficacy and less severe adverse effects than methotrexate or azathioprine. Since the patient in the present case was at risk of renal dysfunction due to severely impaired cardiac function, we decided to use MMF in combination with corticosteroids as a second-line therapy for refractory CS.

The data regarding the use of MMF in sarcoidosis, especially in $\mathrm{CS}$, are limited. The present report suggests that MMF may be safe and effective in suppressing CS disease activity and refractory VT, although it is difficult to perfectly prevent physiological FDG uptake in the normal and failing myocardium. More data with regard to MMF therapy for CS are needed to establish this strategy for refractory CS.

\section{Conclusions}

We report the efficacy of combination therapy with corticosteroids and MMF in a patient with sarcoidosis and refractory cardiac involvement. MMF, which has fewer adverse effects than methotrexate or azathioprine, should be considered as an 
alternative option for the treatment of corticosteroid-resistant sarcoidosis.

\section{Conflicts of interest}

The authors declare that they have no conflicts of interest.

\section{References}

[1] Iannuzzi MC, Rybicki BA, Teirstein AS. Sarcoidosis. N Engl J Med 2007;357:2153-65.

[2] Yazaki Y, Isobe M, Hiroe M, Morimoto S, Hiramitsu S, Nakano T, Izumi T, Sekiguchi M, Central Japan Heart Study Group. Prognostic determinants of long-term survival in Japanese patients with cardiac sarcoidosis treated with prednisone. Am J Cardiol 2001;88:1006-10.

[3] Kandolin R, Lehtonen J, Airaksinen J, Vihinen T, Miettinen H, Ylitalo K, Kaikkonen K, Tuohinen S, Haataja P, Kerola T, Kokkonen J, Pelkonen M Pietilä-Effati P, Utrianen S, Kupari M. Cardiac sarcoidosis: epidemiology, characteristics, and outcome over 25 years in a nationwide study. Circulation 2015;131:624-32.
[4] Tahara N, Tahara A, Nitta Y, Kodama N, Mizoguchi M, Kaida H, Baba K, Ishibashi M, Hayabuchi N, Narula J, Imaizumi T. Heterogeneous myocardial FDG uptake and the disease activity in cardiac sarcoidosis. JACC Cardiovasc Imaging 2010;3:1219-28

[5] Nagai S, Yokomatsu T, Tanizawa K, Ikezoe K, Handa T, Ito Y, Ogino S, Izumi T. Treatment with methotrexate and low-dose corticosteroids in sarcoidosis patients with cardiac lesions. Intern Med 2014;53:427-33.

[6] Shimizu H, Takahashi M, Takeda S, Inoue S, Fujishiro J, Hakamata Y, Kaneko T, Murakami T, Takeuchi K, Takeyoshi I, Morishita Y, Kobayashi E. Mycophenolate mofetil prevents transplant arteriosclerosis by direct inhibition of vascular smooth muscle cell proliferation. Transplantation 2004;77:1661-7.

[7] Dandel M, Jasaityte R, Lehmkuhl H, Knosalla C, Hetzer R. Maintenance immunosuppression with mycophenolate mofetil: long-term efficacy and safety after heart transplantation. Transplant Proc 2009;41:2585-8.

[8] Brill AK, Ott SR, Geiser T. Effect and safety of mycophenolate mofetil in chronic pulmonary sarcoidosis: a retrospective study. Respiration 2013;86: 376-83.

[9] Vorselaars AD, Wuyts WA, Vorselaars VM, Zanen P, Deneer VH, Veltkamp M, Thomeer M, van Moorsel CH, Grutters JC. Methotrexate vs azathioprine in second-line therapy of sarcoidosis. Chest 2013;144:805-12.

[10] Allison AC, Eugui EM. Mycophenolate mofetil and its mechanisms of action. Immunopharmacology 2000;47:85-118. 